\title{
Erfinder des Alltags
}

Erhard Taverna

erhard.taverna[at]saez.ch
Erfinder beflügeln die Fantasie. Sie heben die Welt aus den Angeln, sie retten die Menschheit oder schaffen Monster, die wir nicht mehr loswerden. Die Realität ist zum Glück weniger spektakulär, aber nicht weniger wirksam, wie der Earl von Sandwich oder das Fladenbrot von Kadir Nurmann beweisen. Nimmt man das Europäische Patentamt als Gradmesser der Innovationslust, dann war 2012 ein Rekordjahr mit über einer Viertelmillion Patentanmeldungen.

Die lange Liste der Erfindungen weist auch viele Mediziner auf, die auf ihrem Fachgebiet Neues, wie den Herzkatheter (Werner Forstmann), die erste gläserne Kontaktlinse (Eugen Fick), die Marknagelung (Gerhard Küntscher), das erste Antibiotikum (Alexander Fleming) oder das erste brauchbare EEGGerät (Hans Berger) entwickelt haben. Nicht alle erfuhren die gebührende Anerkennung. Eine brillante Idee zur falschen Zeit am falschen Ort kann Kopf und Kragen kosten. Ludwig Haberlandt (1885-1932), ein österreichischer Pionier der hormonalen Kontrazeption, wurde aus seinem Land gemobbt und in den Suizid getrieben. Der aus Wien vertriebene Chemiker Carl Djerassi hat Haberlandt stets als «Vater» und sich selber als «Mutter der Antibabypille» bezeichnet.

Es kommt zuweilen vor, dass Ärzte, auch ausserhalb ihres eigentlichen Fachgebietes, kreativ die Gesellschaft bereichern. Allerdings immer hart bedrängt von Apothekerkollegen, die mit Rezepturen für Coca-Cola, Gummibärchen und Zahnpasta die Welt irreversibel verändert haben. Lässt man die frühen Alchimistenärzte aussen vor, dann wird man schnell einmal in der Renaissance fündig. Gerolamo Cardano (1501-1576) war ein scharfsinniger Arzt, Philosoph und Mathematiker, den Könige und Kardinäle sich vergeblich zum Leibarzt wünschten. Cardano wollte unabhängig bleiben. Er entdeckte wichtige Gesetze der Wahrscheinlichkeitsrechnung und

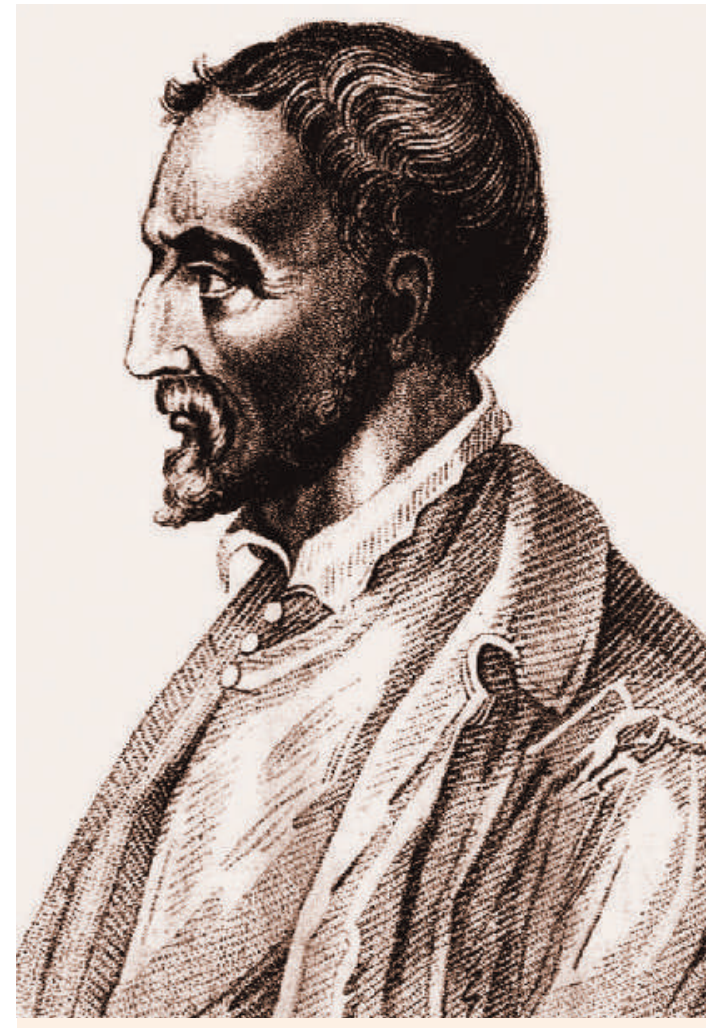

Scharfsinniger Arzt, Philosoph und Mathematiker: Gerolamo Cardano (1501-1576).

Gitters, wird heute im Online-Banking verwendet. Ein Leuchtturm der Wissenschaft, seither kaum mehr zu übertreffen.

Auch nicht vom Stadtphysicus Christoph von Hellwig, der die Planetentafel neu rechnete und 1704 den Hundertjährigen Kalender drucken liess. Ihm als Erfinder der Zahnbürste hat die Stadt Tennstedt ein Denkmal errichtet. Der Schotte William Cullen erfand einen Vorläufer der ICD (International Classification of Diseases) und bastelte mit

\section{Der Schotte William Cullen erfand einen Vorläufer der ICD und bastelte mit verdampfendem Diethyläther die erste Kühlmaschine für} seine anatomischen Präparate.

Kombinatorik, womit er sich bei Glücksspielen sein Gehalt aufbesserte. Die nach ihm benannte Kardanwelle entwarf er für eine Kutsche von Karl V., seine Verschlüsselungstechnik, das Prinzip des Cardan- verdampfendem Diethyläther die erste Kühlmaschine für seine anatomischen Präparate. Beides, die Taxonomie und das konservierende Eis, sind nicht mehr wegzudenken. Ganz im Gegensatz zur Appara- 

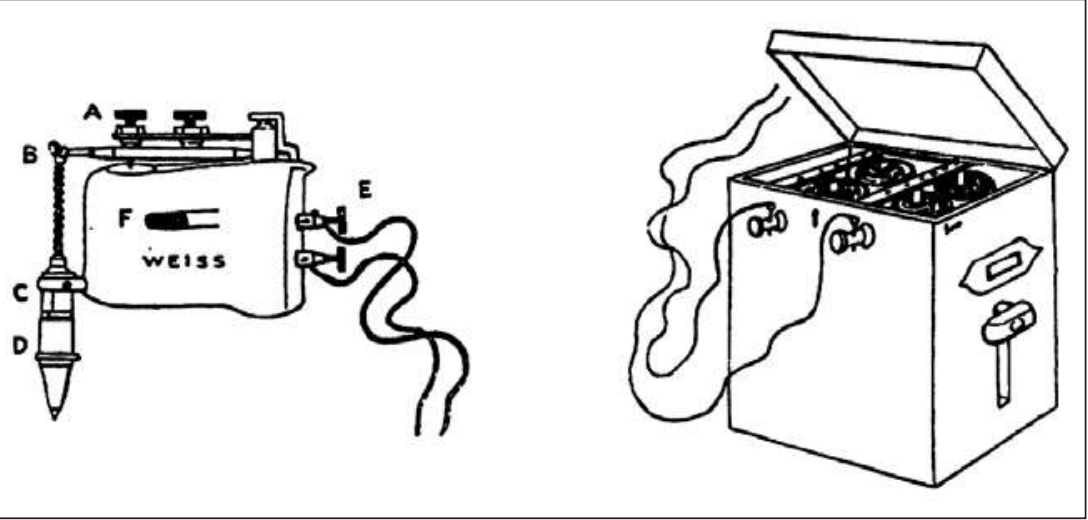

Erfinder des «Vibrators»: Illustration aus Joseph Mortimer Granvilles Schrift Nerve-vibration and excitation as agents in the treatment of functional disorder and organic disease (London 1883).

tur eines weiteren Zeitgenossen, denn auch gutgemeinte Absichten können leicht durch Missbrauch diskreditiert werden. Das musste besonders der unglückliche Joseph Ignace Guillotin erfahren, obwohl seine Maschine die Justiz bis in unsere Tage bereichert hat.

Weniger hart gebettet liegt der Fall von Victor von Bruns. Erfolgreicher Chirurg und Begründer der modernen Laryngologie in Tübingen, wurde er Ehren- schenk, das gemäss Werbung den Frauen «glänzende Augen und rosige Wangen» bescherte. Die Filmstory «Hysteria» (deutsch: In guten Händen) von 2011 schildert komödiantisch, wie die viktorianische Prüderie ins Vibrieren kam.

Die Filmgeschichte hat sich auch John Harvey Kelloggs bemächtigt, der mit seinem Bruder die Cornflakes und Erdnussbutter entwickelte. Als Ernährungs- und Moralapostel hat er, wie die ähnlich denkenden Kollegen Oskar Bircher-Benner und Werner Kollath, die Essgewohnheiten von ganzen Nationen nachhaltig verändert.

Soll man den Schrebergarten des Hamburger Praktikers Daniel Gottlob Schreber auch zu den zivilisatorischen Errungenschaften zählen? Immerhin ist sein Beitrag zur städtebaulich unübersehbaren Randzone umstritten. Unbestritten ist hingegen das Heilmittel zum Weltfrieden, das der polnische Augenarzt Ludwik Lejzer Zamenhof mit seinem Esperanto erfand. Seine weniger bekannte Menschheitslehre versuchte die Probleme einer multikulturellen Gesellschaft zu lösen. Eine grosse Gemeinde pflegt seine Kunstsprache weiter. Strassennamen, Denkmäler und Briefmarken in der ganzen Welt gedenken seiner.

Welcher Mediziner gehört noch in die Ruhmeshalle praktischer Alltagserfindungen? Sicher wäre

\section{«In das Pantheon der nützlichen Alltagsdinge befördert ihn sein \\ Verfahren zur Herstellung von Verbandwatte, der guten, alten, saugfähigen Dr. v. Bruns-Watte.»}

bürger und für seine fachlichen Verdienste mit dem Ritterkreuz ausgezeichnet. In das Pantheon der nützlichen Alltagsdinge befördert ihn sein Verfahren zur Herstellung von Verbandwatte, der guten, alten, saugfähigen Dr. v. Bruns-Watte. Einen gleichwertigen Platz wollen wir dem schottischen Arzt Neil Arnott zugestehen, der seine Weltneuheit Wasserbett nebst Ventilator und Ofen kreierte.

Mit der Industrialisierung im 19. und 20. Jahrhundert nimmt die Zahl der Erfindungen exponentiell zu. Ingenieure, Chemiker und Physiker bestimmen das Tempo der Innovationen. Mühelos mithalten kann der Erfinder des elektrischen Vibrators, der englische Arzt Joseph Mortimer Granville. Bekannt als «Granvilles Hammer», mit mehreren auswechselbaren Aufsätzen, begann die Erfolgsgeschichte des surrenden Freudenspenders. Das Gerät war ursprünglich dazu gedacht, die verspannten Muskeln männlicher Patienten zu lockern. Als Wunderwaffe gegen weibliche Hysterie, Haarausfall und Hüftspeck geriet es in den 1920er Jahren zum Weihnachtsge- noch der Walliser Dr. med. Guglielminetti zu erwähnen, der in Monaco erstmals Theorie und Praxis von der Oberflächenteerung entwickelte. Mit dem globalen Netz der Asphaltstrassen hat er seinen Zunamen «Docteur Goudron» wahrlich verdient. Beinahe könnte man auch Laszlo Jozsef Biro, den ungarischen Erfinder des Kugelschreibers, auf das Podest heben. Er hat immerhin einige Semester Medizin studiert. Als letzter Erfinder darf in dieser Aufzählung auch Gunther von Hagens nicht vergessen gehen. Den von manchen verliehenen Ehrentitel «Robin Hood der Anatomie» hat er redlich verdient. Plastination sei Dank haben wir endlich unsere Verwesung mit flüssigem Silikonkautschuk überwunden.

Alle erwähnten und unerwähnten Helden seien Kolleginnen und Kollegen zur Nachahmung empfohlen. Viele Wege führen zum Ruhm. In Hamburg erwartet sie ein anspornender Wettbewerb, der mit dem Daniel Düsentrieb Preis auch 2013/2014 die glücklichen Kandidaten belohnt. 